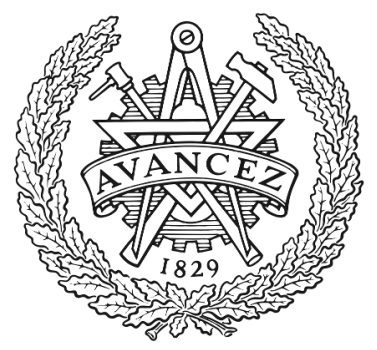

CHALMERS

UNIVERSITY OF TECHNOLOGY

\title{
When to Use Optical Amplification in Noncoherent Transmission: An Information-Theoretic Approach
}

Downloaded from: https://research.chalmers.se, 2023-04-26 15:37 UTC

Citation for the original published paper (version of record):

Keykhosravi, K., Agrell, E., Secondini, M. et al (2020). When to Use Optical Amplification in Noncoherent Transmission: An Information-Theoretic Approach. IEEE Transactions on

Communications, 68(4): 2438-2445. http://dx.doi.org/10.1109/TCOMM.2020.2968890

N.B. When citing this work, cite the original published paper.

(C2020 IEEE. Personal use of this material is permitted.

However, permission to reprint/republish this material for advertising or promotional purposes 


\title{
When to Use Optical Amplification in Noncoherent Transmission: An Information-Theoretic Approach
}

\author{
Kamran Keykhosravi, Student Member, IEEE, Erik Agrell, Fellow, IEEE, \\ Marco Secondini, Senior Member, IEEE, and Magnus Karlsson, Fellow, OSA.
}

\begin{abstract}
The standard solution for short-haul fiberoptic communications is to deploy noncoherent systems, i.e., to modulate and detect only the light intensity. In such systems, the signal is corrupted with optical noise from amplifiers and with thermal (electrical) noise. The capacity of noncoherent optical links has been studied extensively in the presence of either optical noise or thermal noise. In this paper, for the first time, we characterize the capacity under an average power constraint with both noise sources by establishing upper and lower bounds. In the two extreme cases of zero optical noise or zero thermal noise, we assess our bounds against some well-known results in the literature; improvements in both cases are observed. Next, for amplified fiber-optic systems, we study the trade-off between boosting signal energy (mitigating the effects of thermal noise) and adding optical noise. For a wide spectrum of system parameters and received power levels, we determine the optimal amplification gain. While mostly either no amplification or high-gain amplification is optimal, the best performance is for some parameter intervals achieved at finite gains.
\end{abstract}

Index Terms - Channel capacity, noncoherent optics, optical amplification, passive optical network, phasenoise channel.

\section{INTRODUCTION}

$\mathbf{N}$ ONCOHERENT transmission systems have been extensively investigated as a low-cost solution to short-haul optical communication. These systems are generally based on intensity modulation and direct detection (IMDD), where the information is conveyed via the optical signal intensity. At the transmitter, information bits are mapped to a nonnegative signal, which modulates the light intensity. At the receiver, the demodulation is performed by transforming the optical intensity to an electrical current via a photodetector. IMDD systems have applications in both wireless optical communications [1] and fiber optics (e.g., passive optical networks and

This work was supported by the Swedish Research Council (VR) under Grants 2013-5271 and 2017-03702, the Knut and Alice Wallenberg (KAW) Foundation under Grant 2013.0021, S o KG Eliassons minnesfond, and Chalmers Research Foundation.

K. Keykhosravi and E. Agrell are with the Department of Electrical Engineering, and Magnus Karlsson is with the Department of Microtechnology and Nanoscience, all at Chalmers University of Technology, 41296 Gothenburg, Sweden (e-mail: agrell@chalmers.se).

M. Secondini is with the TeCIP Institute, Scuola Superiore Sant'Anna, 56124 Pisa, Italy. optical interconnection). The focus of this paper is on the latter one; however, we use some relevant literature from the wireless optical field to assess our results, which are relevant also for some free-space optical scenarios [2].

In many practical cases, the performance of IMDD systems is ultimately limited by the thermal (electrical) noise of the receiver. This is the case, for instance, when shot noise is negligible (e.g., with PIN photodetectors [3]) and the main effect of channel propagation is signal attenuation (e.g., short-haul or long-haul dispersionmanaged optical fiber links and free-space optical links with quasi-static fading [2]). In such a scenario, optical amplification can be used to boost the optical power before photodetection, hence increasing the signal-to-thermalnoise ratio. However, besides its additional cost, optical amplification also introduces some optical noise, whose impact depends in general on the amplification gain. Therefore, it is essential to quantify the actual benefits (if any) of using optical amplification and to determine what is the optimal amplification gain.

While both the electrical and optical noise are commonly modeled as additive white Gaussian noise (AWGN) in their respective domains, the presence of a squarelaw detector (the photodetector) between the two noise sources makes the overall channel non-AWGN. Thus, the optimization of the amplification gain cannot be based on a simple analysis of the overall signal-to-noise ratio (SNR), as the system performance depends in a nontrivial way on the actual noise statistics (which change with the gain) and on the adopted modulation format and detection strategy. In fact, in the current era of lowcost and nearly-ubiquitous digital signal processing [4], it makes more sense to consider an optimal scenario, in which both modulation and detection are tailored to the channel statistics.

In this work, we base the aforementioned analysis on information-theoretic grounds, studying channel capacity in the presence of both optical and thermal noise and investigating its dependence on the amplifier gain. We consider a discrete-time channel model derived under the assumptions of symbol-time sampling and no intersymbol interference (ISI). As discussed above, this model is of practical relevance when a direct-detection thermal-noiselimited receiver is considered and the main effect of channel propagation is signal attenuation, which may apply 
to both optical fiber and free-space optics scenarios. The impact of ISI and the potential benefits of oversampling [5] in this scenario are left for future research.

In the most general case of IMDD systems, the received signal is corrupted with amplification noise in the optical domain and with thermal noise in the electrical one. Furthermore, optical and electrical filters as well as fiber dispersion can induce ISI. The performance analyses of such systems (with ISI) are limited to numerical calculation of either bit error rate [6] or achievable information rate (AIR) [7]. In this paper, we consider ISI-free communication using advanced signaling [8]-[11] over a fiberoptical system with negligible dispersion (due to short fiber length or the use of dispersion-compensation fibers).

The capacity of ISI-free IMDD fiber-optic channel models has been extensively studied in the literature in two main cases [12, Ch. 11], namely, optical-noise-dominated and thermal-noise-dominated systems. With the former class, the thermal noise is neglected and the optical noise is added to the signal before the photodetector. The resulting discrete-time channel model is [12, Eq. (11.23)] [13]-[15]

$$
\mathbf{y}=\left|\mathbf{x}+\mathbf{n}_{\mathrm{o}}\right|^{2}
$$

where $\mathbf{x}$ and $\mathbf{y}$ denote the real, nonnegative transmitted and received symbols, respectively. The optical noise $\mathbf{n}_{\mathrm{o}} \in \mathbb{C}$ follows a circularly-symmetric Gaussian distribution. The capacity is lower-bounded in [13] using a halfnormal distribution and is approximated via numerical optimization in [15]. Furthermore, the channel (1) has been studied in the context of a phase-noise channel in [16]-[18], where upper and lower bounds on the capacity have been established.

With thermal-noise-dominated IMDD fiber-optic systems, the optical noise is assumed negligible and the input-output relation can be described as [12, Eq. (11.53)] $[11],[19]-[22]$

$$
\mathbf{y}=\mathbf{x}^{2}+\mathbf{n}_{\mathrm{t}}
$$

where the thermal noise $\mathbf{n}_{\mathrm{t}}$ is modeled as a zero-mean real Gaussian random variable. The input symbol $\mathbf{x}$ is positive but the channel output $\mathbf{y}$ may take negative values. In the context of wireless optics, bounds on the capacity of (2) were developed in [23], which are tight in the high and low power regimes. In [24], [25], the capacity was numerically evaluated and capacity bounds were derived. The capacity of multi-subcarrier systems was studied in [19], [21]. Also, the capacity of multiple-input multipleoutput and multiple-input single-output wireless optical channels was studied in [26]-[29].

In this paper, we study the capacity of an IMDD channel with both optical and thermal noises, whose input-output relation is described as

$$
\mathbf{y}=\left|\mathbf{x}+\mathbf{n}_{\mathrm{o}}\right|^{2}+\mathbf{n}_{\mathrm{t}}
$$

under an average power constraint. To the best of our knowledge, this capacity has not been investigated before.

The channel model (3) applies to all optical links with optical amplifiers, with some caveats. For example, optical noise-noise beating from the polarization orthogonal to the signal is neglected. Even if it, in principle, can be included in $\mathbf{n}_{\mathrm{t}}$ at the expense of making $\mathbf{n}_{\mathrm{t}}$ non-Gaussian, it is small relative to the copolarized noise-signal beating in most systems of practical interest. Shot noise is assumed to be negligible relative to thermal noise, which applies to most practical systems as well. Intersymbol inteference from, e.g., chromatic or polarization-mode dispersion is assumed to be negligible or optically compensated, which is reasonable and applies to most IMDD links based on single-mode fiber. Other practical impairments such as channel crosstalk, bandwidth limitations and nonlinear distortions from, e.g., lasers, detectors, amplifiers, and fibers are less fundamental, and are neglected for simplicity. Thus, the model (3) does account for the relevant fundamental noise sources in most IMDD links, including freespace optical links with optically preamplified receivers.

This paper comprises two parts. In the first part, we derive a lower bound on the capacity of (3) by numerically calculating the mutual information (MI). Also, an upper bound is derived based on the duality technique, which was proposed in [30] and used in a number of subsequent papers, e.g., [17], [23], [26], [27]. For the two special cases of optical-noise-dominated $\left(\mathbf{n}_{\mathrm{t}}=0\right)$ and thermal-noisedominated $\left(\mathbf{n}_{\mathrm{o}}=0\right)$ systems, we compare our bounds to the ones in [16] and [23], respectively. We show that at the cost of increased computational complexity, our bounds improve on the results of [16], [23]. Furthermore, we characterize the capacity of the general case (3) with nonzero thermal and optical noises using our upper and lower bounds. By calculating capacity approximations using the Blahut-Arimoto algorithm (BAA) [31], [32] we show that our lower bound follows the capacity closely.

In IMDD systems, at low received signal powers, optical amplification is deployed to mitigate the effects of thermal noise. At high powers, however, amplifying the signal degrades the SNR. In the second part of the paper, for a large spectrum of channel parameters and received signal powers, we use our capacity lower bound to determine whether optical amplification improves the capacity or not. We observed that while for a wide range of parameters either no amplification or high-gain amplification is optimal, within some parameter intervals and received powers, amplification with finite gains maximizes the capacity. We also study the AIRs using pulse-amplitude modulation (PAM) and compare the results with our capacity lower bound.

The remainder of this paper is organized as follows. In Section II, we investigate the optical and thermal noise variances in the channel model (3). Upper and lower bounds are presented and numerically evaluated in Section III. Section IV studies the capacity of amplified noncoherent systems, where optimal amplification gains for a large spectrum of channel parameters are determined. Finally, conclusions are drawn in Section V.

Notation: We use bold-face letters to denote random quantities. Expectation over the random variables $\mathbf{x}$ is represented by $\mathrm{E}_{\mathbf{x}}[\cdot]$. The imaginary unit is indicated by 
$j=\sqrt{-1}$. The probability distribution function of the random variable $\mathbf{x}$ is denoted by $p_{\mathbf{x}}$ and its characteristic function by $\Phi_{\mathbf{x}}(t)=\mathrm{E}\left[e^{j t \mathbf{x}}\right]$. Entropy and differential entropy are denoted by $H(\cdot)$ and $h(\cdot)$, respectively. $I(\mathbf{x} ; \mathbf{y})$ represents the MI between two random variable $\mathbf{x}$ and $\mathbf{y}$. The real Gaussian distribution with mean $\mu$ and variance $\sigma^{2}$ is denoted by $\mathcal{N}\left(\mu, \sigma^{2}\right)$ and the circularly symmetric complex Gaussian distribution by $\mathcal{C N}\left(0, \sigma^{2}\right)$. All logarithms are in base two.

\section{Description OF THE NOISE SOURCES}

As mentioned in Section I, in this paper, we investigate the capacity of the noncoherent optical channel model in (3). The capacity of this channel under the average power constraint $P$ can be determined by $[33]$

$$
\mathcal{C}=\max _{p_{\mathbf{x}}} I(\mathbf{x} ; \mathbf{y})
$$

where the maximum is taken over all distributions $p_{\mathbf{x}}$ that satisfy $\mathrm{E}\left[\mathrm{x}^{2}\right] \leq P$. Since $\mathrm{MI}$ is preserved under one-to-one transformations of $\mathbf{y}$, one can observe that the capacity may be expressed as a function of two parameters, namely, signal-to-thermal-noise ratio

$$
\eta=P / \sqrt{P_{\mathrm{t}}}
$$

and thermal-to-optical-noise ratio

$$
\zeta=\sqrt{P_{\mathrm{t}}} / P_{\mathrm{o}}
$$

where $P_{\mathrm{t}}$ (in ampere-squared) and $P_{\mathrm{o}}$ (in ampere) are thermal and optical noise variances, respectively, which will be further discussed below.

The receiver thermal noise $\mathbf{n}_{\mathrm{t}} \sim \mathcal{N}\left(0, P_{\mathrm{t}}\right)$ originates from the random movement of electrons at any nonzero temperature [34, Sec. 4.4]. It has a variance of $P_{\mathrm{t}}=$ $2 N_{\mathrm{t}} W_{\mathrm{t}}$, where $N_{\mathrm{t}}$ denotes the noise two-sided spectral density, $W_{\mathrm{t}}$ the noise bandwidth or electrical filter bandwidth (in hertz). The thermal noise spectral density is calculated as [34, Eq. (4.4.7)] $N_{\mathrm{t}}=2 k_{\mathrm{b}} T / R_{\mathrm{L}}$ (in ampere-squaredsecond), where $k_{\mathrm{b}}=1.38 \cdot 10^{-23}$ (joule per kelvin) is Boltzman's constant, $T$ is the temperature in kelvin, and $R_{\mathrm{L}}$ is the receiver resistance in ohms.

The main source of optical noise in systems with optical amplification is the amplified spontaneous emission (ASE) noise [35]. A lumped phase-insensitive fiber amplifier with power gain $G$ injects an optical noise to the signal. This noise can be modeled as an additive circularly-symmetric white Gaussian process. To model this noise properly as in (3), its variance should be multiplied by the photodiode responsivity $R$ (in ampere per watt) to make its unit consistent with the thermal noise ${ }^{1}$. Then, we have $\mathbf{n}_{\mathrm{o}} \sim$ $\mathcal{C N}\left(0, P_{\mathrm{o}}\right)$ with $P_{\mathrm{o}}=(G-1) \tilde{P}_{\mathrm{o}}$, where $\tilde{P}_{\mathrm{o}}=R h \nu n_{\mathrm{sp}} W_{\mathrm{o}}$ (in ampere). Here, $h \nu$ is the optical photon energy (in joule), $n_{\mathrm{sp}}$ is the spontaneous emission factor, and $W_{\mathrm{o}}$ is the optical noise (or filter) bandwidth (in hertz). The photodiode responsivity can be written as [34, Eq. (4.1.3)]

\footnotetext{
${ }^{1}$ Also, the power constraint $P$ (in ampere) is calculated by multiplying an optical power constraint (in watt) by $R$ (in ampere per watt).
}

$R=\xi q / h \nu$, where $\xi$ is quantum efficiency and $q$ is the electron's charge. Therefore, $\tilde{P}_{\mathrm{o}}$ can be rewritten as $\tilde{P}_{\mathrm{o}}=\xi q n_{\mathrm{sp}} W_{\mathrm{o}}$. Note that with no amplification $(G=1)$, no ASE noise is added to the signal.

The channel model (3) can be rewritten to account for the amplification gain as

$$
\mathbf{y}=\left|\sqrt{G} \tilde{\mathbf{x}}+\sqrt{G-1} \tilde{\mathbf{n}}_{\mathrm{o}}\right|^{2}+\mathbf{n}_{\mathrm{t}}
$$

where $\tilde{\mathbf{n}}_{\mathrm{o}} \sim \mathcal{C} \mathcal{N}\left(0, \tilde{P}_{\mathrm{o}}\right)$ and $\tilde{\mathbf{x}}$ is the received signal before amplification. The capacity of (7) under the power constraint $\mathrm{E}\left[|\tilde{\mathbf{x}}|^{2}\right] \leq \tilde{P}$ is equal to the capacity of (3) with $P=G \tilde{P}$ and $P_{\mathrm{o}}=(G-1) \tilde{P}_{\mathrm{o}}$. Consequently, the capacity can be expressed as a function of amplification gain $G$, nonamplified signal-to-thermal-noise ratio

$$
\tilde{\eta}=\tilde{P} / \sqrt{P_{\mathrm{t}}}
$$

and thermal-to-nonamplified-optical-noise ratio

$$
\begin{aligned}
\tilde{\zeta} & =\sqrt{P_{\mathrm{t}}} / \tilde{P}_{\mathrm{o}} \\
& =\frac{\sqrt{4 k_{\mathrm{b}} T W_{\mathrm{t}}}}{\xi q n_{\mathrm{sp}} W_{\mathrm{o}} \sqrt{R_{\mathrm{L}}}} .
\end{aligned}
$$

In a generic noncoherent optical system, $\eta(5)$ and $\zeta$ (6) depend on many parameters, e.g., the transmitted power, signal bandwidth, fiber loss, transmission length, and parameters of the transmitter, receiver, and amplifier. Apart from the description provided in this section, nonlinear inter- and intrachannel crosstalk may contribute to the optical noise; also insertion losses and electrical amplification may influence $\eta$ and $\zeta$. In this paper, we take $\eta$ and $\zeta$ as free parameters and evaluate the capacity of (3) for a large range of these parameters in Section III-C. Also, to study the influence of amplification gain $G$ on the capacity, in Section IV we sweep over a large range of $\tilde{\eta}$ (8) and $\tilde{\zeta}(9)$.

\section{CAPACITY BOUNDS}

Since $\mathbf{x} \geq 0$, we have $I(\mathbf{x} ; \mathbf{y})=I\left(\mathbf{x}^{2} ; \mathbf{y}\right)$. Therefore, the capacity (4) can be expressed in terms of $\mathbf{u}=\mathbf{x}^{2}$ as

$$
\begin{aligned}
\mathcal{C} & =\max _{p_{\mathbf{u}}} I(\mathbf{u} ; \mathbf{y}) \\
& =\max _{p_{\mathbf{u}}}(h(\mathbf{y})-h(\mathbf{y} \mid \mathbf{u}))
\end{aligned}
$$

with the power constraint $\mathrm{E}[\mathbf{u}] \leq P$. In Sections III-A and III-B, we present a lower bound and an upper bound on the capacity, respectively. In Section III-C, we provide a numerical evaluation of these bounds.

\section{A. Gamma-distribution lower bound}

A lower bound on the capacity can be obtained by calculating $h(\mathbf{y} \mid \mathbf{u})$ and $h(\mathbf{y})$ for a fixed $p_{\mathbf{u}}$. To do so, one should obtain $p_{\mathbf{y} \mid \mathbf{u}=u}$ for any $u \geq 0$ and also $p_{\mathbf{y}}$. The former can be calculated by noting that $\Phi_{\mathbf{y} \mid \mathbf{u}=u}$ (the characteristic function of $\mathbf{y} \mid \mathbf{u}=u$ ) is obtained by multiplying the 
characteristic function of a noncentral chi-squared random variable with two degrees of freedom by $\Phi_{\mathbf{n}_{t}}$. Specifically,

$$
\begin{aligned}
p_{\mathbf{y} \mid \mathbf{u}=u}(y) & =\frac{1}{2 \pi} \int_{-\infty}^{\infty} \Phi_{\mathbf{y} \mid \mathbf{u}=u}(t) e^{-j t y} d t \\
\Phi_{\mathbf{y} \mid \mathbf{u}=u}(t) & =\Phi_{\left|\sqrt{u}+\mathbf{n}_{\mathrm{o}}\right|^{2}}(t) \Phi_{\mathbf{n}_{\mathrm{t}}}(t) \\
& =\exp \left(\frac{j u t}{1-j P_{\mathrm{o}} t}-\frac{P_{\mathrm{t}} t^{2}}{2}\right) /\left(1-j P_{\mathrm{o}} t\right)
\end{aligned}
$$

where (14) follows from (3) and the fact that $\mathbf{n}_{\mathrm{o}}$ and $\mathbf{n}_{\mathrm{t}}$ are independent. To reduce the computational complexity, (13) can be calculated via the fast Fourier transform (FFT).

The output probability density function can be calculated as

$$
p_{\mathbf{y}}(y)=\int p_{\mathbf{y} \mid \mathbf{u}=u}(y) p_{\mathbf{u}}(u) \mathrm{d} u .
$$

By substituting (13) into (16), after some algebraic steps,

$$
\begin{aligned}
& p_{\mathbf{y}}(y)=\frac{1}{2 \pi} \int_{-\infty}^{\infty} \Phi_{\mathbf{y}}(t) e^{-j t y} \mathrm{~d} t \\
& \Phi_{\mathbf{y}}(t)=\frac{e^{-P_{\mathrm{t}} t^{2} / 2}}{1-j P_{\mathrm{o}} t} \Phi_{\mathbf{u}}\left(\frac{t}{1-j P_{\mathrm{o}} t}\right) .
\end{aligned}
$$

Therefore, $p_{\mathbf{y}}$ can be calculated by (17) via the FFT. The lower bound considered in this paper is based on the gamma distribution with parameters $\alpha>0$ and $\beta=\alpha / P$, whose characteristic function is

$$
\Phi_{\mathbf{u}}(t)=(1-j t P / \alpha)^{-\alpha} .
$$

The lower bound is obtained by maximizing $I(\mathbf{u} ; \mathbf{y})=$ $h(\mathbf{y})-h(\mathbf{y} \mid \mathbf{u})$ over the parameter $\alpha$, where $h(\mathbf{y} \mid \mathbf{u})$ and $h(\mathbf{y})$ are numerically calculated based on (13)-(15) and (17)-(18), respectively.

\section{B. Generalized-gamma-distribution upper bound}

In this section, we use the duality technique [30] with a generalized-gamma-distribution auxiliary output distribution to upper-bound the capacity. We begin by upperbounding $h(\mathbf{y})$. Fix a constant $a \in \mathbb{R}$ and let $\tilde{\mathbf{y}}=\mathbf{y}+a$. Also, define $\tilde{\mathbf{r}}=|\tilde{\mathbf{y}}|$ and $\tilde{\boldsymbol{\theta}}=\operatorname{sign}(\tilde{\mathbf{y}})$. Since differential entropy is invariant to translations,

$$
\begin{aligned}
h(\mathbf{y}) & =h(\tilde{\mathbf{y}}) \\
& =h(\tilde{\mathbf{r}})+H(\tilde{\boldsymbol{\theta}} \mid \tilde{\mathbf{r}}) \\
& \leq h(\tilde{\mathbf{r}})+H(\tilde{\boldsymbol{\theta}})
\end{aligned}
$$

where (21) follows from substituting $f_{\tilde{\mathbf{y}}}(\tilde{y})=$ $f_{\tilde{\mathbf{r}}}(|\tilde{y}|) P(\tilde{\theta}=\operatorname{sign}(\tilde{y})|\tilde{\mathbf{r}}=| \tilde{y} \mid)$ into $h(\tilde{\mathbf{y}})=-\mathrm{E}\left[\log f_{\tilde{\mathbf{y}}}(\tilde{\mathbf{y}})\right]$. Using the nonnegativity of relative entropy [36, Thm. 8.6.1] (see also [37, Eq. (92)]) we have

$$
\begin{aligned}
h(\tilde{\mathbf{r}}) & \leq-\mathrm{E}\left[\log \left(q_{\tilde{\mathbf{r}}}(\tilde{\mathbf{r}})\right)\right] \\
& \left.=-\mathrm{E}_{\mathbf{u}}\left[\mathrm{E}\left[\log \left(q_{\tilde{\mathbf{r}}}(\tilde{\mathbf{r}})\right) \mid \mathbf{u}\right]\right]\right]
\end{aligned}
$$

where $q_{\tilde{\mathbf{r}}}$ is an arbitrary distribution over $[0, \infty)$ and $(24)$ follows from the tower rule of expectation. We let $q_{\tilde{\mathbf{r}}}$ be the generalized gamma distribution, i.e.,

$$
q_{\tilde{\mathbf{r}}}(\tilde{r})=\frac{p \tilde{r}^{d-1} e^{-(\tilde{r} / \alpha)^{p}}}{\alpha^{d} \Gamma(d / p)}
$$

with parameters $d>0, p>0$, and $\alpha>0$. We select $\alpha$ such that

$$
\int_{0}^{\infty} \tilde{r} q_{\tilde{\mathbf{r}}}(\tilde{r}) \mathrm{d} \tilde{r}=\mathrm{E}\left[\left|\mathbf{x}+\mathbf{n}_{\mathrm{o}}\right|^{2}\right]+\mathrm{E}\left[\left|\mathbf{n}_{\mathrm{t}}\right|\right]+|a|
$$

where the right hand side of (26) is an approximation of $\mathrm{E}[|\tilde{\mathbf{y}}|]$. This leads to

$$
\alpha=\left(P+P_{\mathrm{o}}+\sqrt{2 P_{\mathrm{t}} / \pi}+|a|\right) \frac{\Gamma(d / p)}{\Gamma((d+1) / p)} .
$$

Here, $\Gamma(\cdot)$ denotes the gamma function.

Similarly as in $(24)$, for any $q_{\tilde{\boldsymbol{\theta}}} \in[0,1]$, the term $H(\tilde{\boldsymbol{\theta}})$ can be upper-bounded as

$$
\begin{aligned}
H(\tilde{\boldsymbol{\theta}}) \leq-\mathrm{E}_{\mathbf{u}}[ & \operatorname{Pr}(\tilde{\boldsymbol{\theta}}=1 \mid \mathbf{u}) \log \left(q_{\tilde{\boldsymbol{\theta}}}\right) \\
& \left.+\operatorname{Pr}(\tilde{\boldsymbol{\theta}}=-1 \mid \mathbf{u}) \log \left(1-q_{\tilde{\boldsymbol{\theta}}}\right)\right] .
\end{aligned}
$$

Therefore, by using (22), (24), (25), and (28), we obtain

$$
h(\mathbf{y}) \leq \mathrm{E}_{\mathbf{u}}\left[g\left(a, p, d, q_{\tilde{\boldsymbol{\theta}}}, \mathbf{u}\right)\right]
$$

where

$$
\begin{aligned}
g( & \left.a, p, d, q_{\tilde{\boldsymbol{\theta}}}, \mathbf{u}\right) \\
= & \log \left(\frac{\alpha^{d} \Gamma(d / p)}{p}\right)+\mathrm{E}_{\mathbf{y}}\left[|\mathbf{y}+a|^{p} \mid \mathbf{u}\right] \log e / \alpha^{p} \\
& +(1-d) \mathrm{E}_{\mathbf{y}}[\log (|\mathbf{y}+a|) \mid \mathbf{u}] \\
& -\operatorname{Pr}(\mathbf{y}>-a \mid \mathbf{u}) \log \left(q_{\tilde{\boldsymbol{\theta}}}\right)-\operatorname{Pr}(\mathbf{y}<-a \mid \mathbf{u}) \log \left(1-q_{\tilde{\boldsymbol{\theta}}}\right) .
\end{aligned}
$$

By (29), (12), and using the method of Lagrange multipliers to include the power constraint, we obtain

$$
\begin{aligned}
\mathcal{C} & \leq \max _{p_{\mathbf{u}}} \mathrm{E}_{\mathbf{u}}\left[g\left(a, p, d, q_{\tilde{\boldsymbol{\theta}}}, \mathbf{u}\right)-h(\mathbf{y} \mid \mathbf{u})+\lambda(P-\mathbf{u})\right] \\
& \leq \max _{u \geq 0}\left[g\left(a, p, d, q_{\tilde{\boldsymbol{\theta}}}, \mathbf{u}=u\right)-h(\mathbf{y} \mid \mathbf{u}=u)+\lambda(P-u)\right] .
\end{aligned}
$$

The upper bound is computed numerically by minimizing (32) (through a brute-force search) over $a \in \mathbb{R}, p>0$, $d>0, q_{\tilde{\boldsymbol{\theta}}} \in[0,1]$, and $\lambda \geq 0\left(\alpha\right.$ is calculated $^{2}$ from $\left.(27)\right)$.

\section{Numerical evaluation of capacity bounds}

In this section, we first provide numerical evaluations of the capacity bounds for the two special cases of $\mathbf{n}_{\mathrm{t}}=0$ and $\mathbf{n}_{\mathrm{o}}=0$ and then we study the general case with both noises. Fig. 1 represents some capacity bounds for the case with zero thermal noise $\left(\mathbf{n}_{\mathrm{t}}=0\right)$, which is a reasonable

\footnotetext{
${ }^{2}$ We used the following search space to calculate (32): $-5 \leq a \leq 5$ (100 equi-spaced values), $0<p<2$ (100 equi-spaced values), $0<$ $d<1.1$ (100 equi-spaced values), $0<q_{\tilde{\boldsymbol{\theta}}}<1$ (20 equi-spaced values), and $-70 \mathrm{~dB} \leq \lambda \leq 70 \mathrm{~dB}$ (100 equi-spaced values).
} 


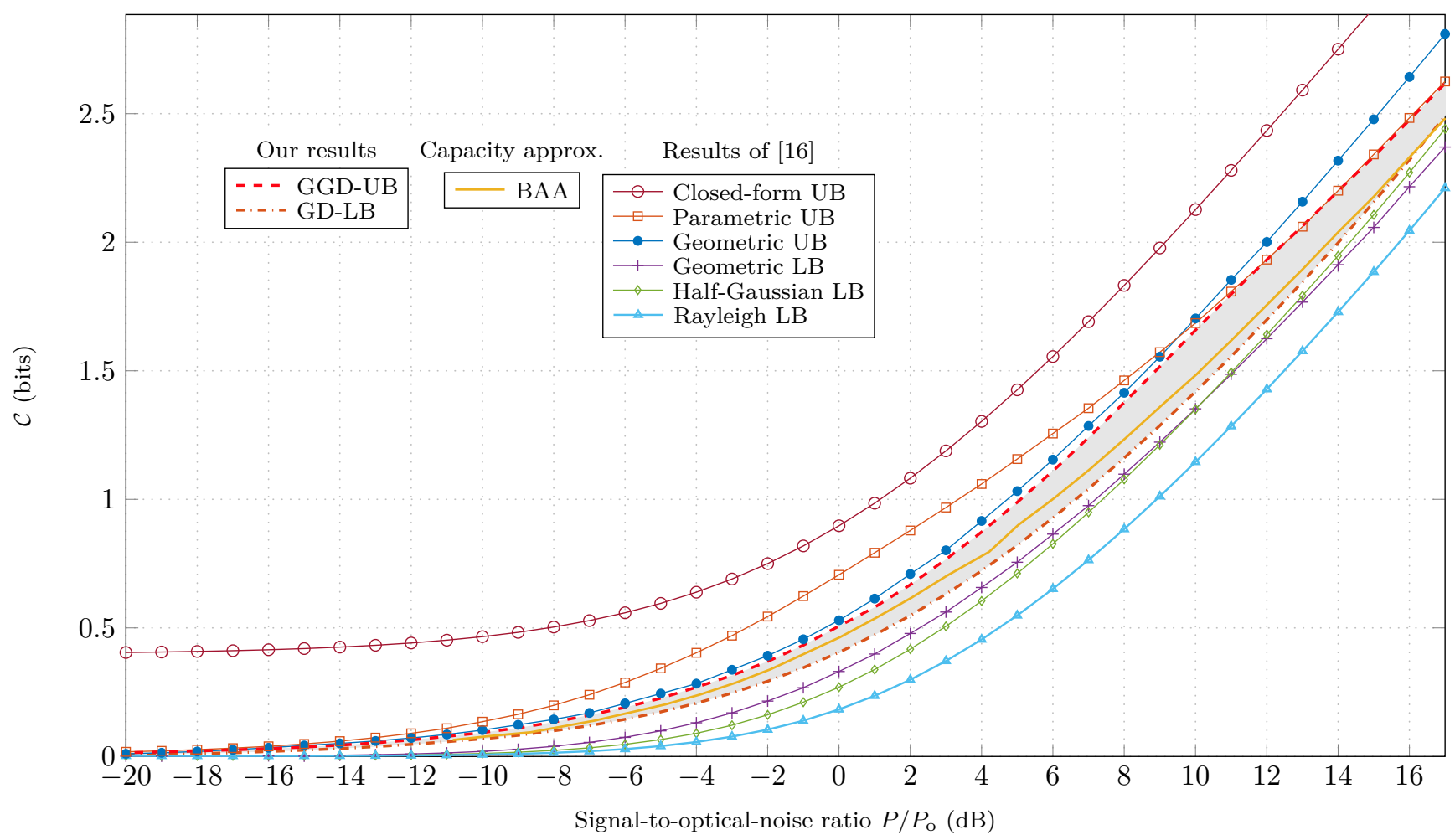

Fig. 1. Bounds on the capacity with $P_{\mathrm{t}}=0$. Our results are compared to the results of [16, Fig. 3]. The shaded area represents the region between the best lower and upper bounds.

approximation for systems with high optical amplification. We compare our bounds with the ones in [16, Fig. 3]. Our results include the gamma-distribution lower bound (GD-LB) in Section III-A and the generalized-gammadistribution upper bound (GGD-UB) in Section III-B. Furthermore, we present a capacity estimate using the Blahut-Arimoto algorithm (BAA) with power constraint [31]. To calculate the GGD-UB, based on our numerical results, with $\mathbf{n}_{\mathrm{t}}=0$, the optimal values for $a$ and $q_{\tilde{\boldsymbol{\theta}}}$ are 0 and 1 , respectively.

It can be concluded from Fig. 1 that the GD-LB improves on the lower bounds in [16], which are based on three distributions over $\mathbf{x}$, namely, Rayleigh, halfGaussian, and geometric distributions. The first two are special cases of the GD-LB with $\alpha=1$ and $\alpha=1 / 2$, respectively. The GGD-UB improves on the upper bounds in [16], which include a closed-form upper bound, a duality upper bound with a geometric-induced distribution of $\mathbf{y}$, and a duality upper bound with a parametric distribution of $\mathbf{y}$. We note that our improvements on the capacity bounds come at the price of increased numerical complexity. The GD-LB needs optimization over the parameter $\alpha$, while no optimization is done with the lower bounds in [16]. Also, the GGD-UB, for $\mathbf{n}_{\mathrm{t}}=0$, is minimized over three parameters, which is one more than the parametric upper bound in [16].

Fig. 2 demonstrates our bounds vs. $\eta$ (5) for the case with $\mathbf{n}_{\mathrm{o}}=0$. Our bounds are compared with the results in [23, Fig. 5]. Notable improvements for the lower bound and slight improvements for the upper bound are obtained,

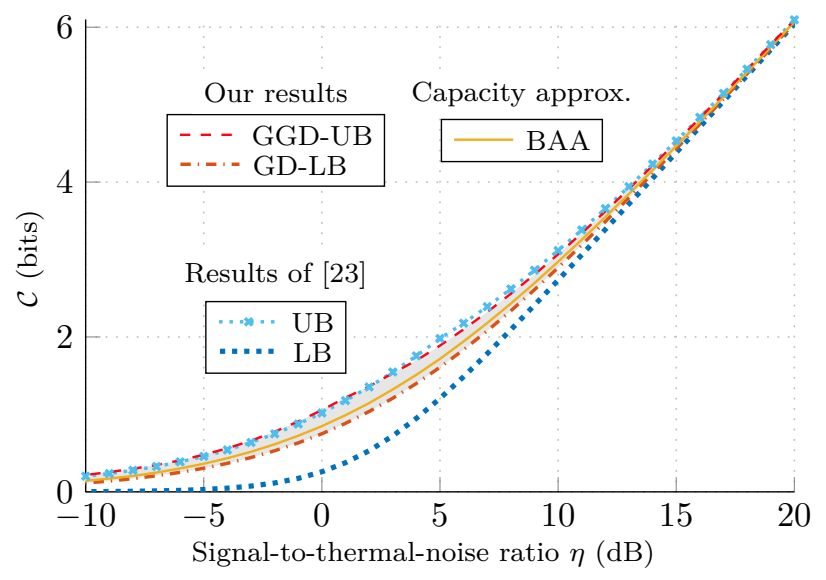

Fig. 2. Bounds on the capacity with $P_{\mathrm{o}}=0$. Our results are compared to the results of [23, Fig. 5].

which are achieved at the cost of increased computational complexity. Also, as can be observed, our lower bound closely follows the capacity approximation obtained via the BAA.

Fig. 3 provides the capacity bounds for three channels with different values of $\zeta(6)$. As is evident, the capacity can be well approximated by the lower and the upper bound. Also, it can be seen that the GD-LB is almost identical to the capacity approximation via the BAA; therefore, it can be used to approximate the capacity with less computational complexity than the BAA. Furthermore, since $\eta \zeta=P / P_{\mathrm{o}}$, the optical signal-to-noise ratio 


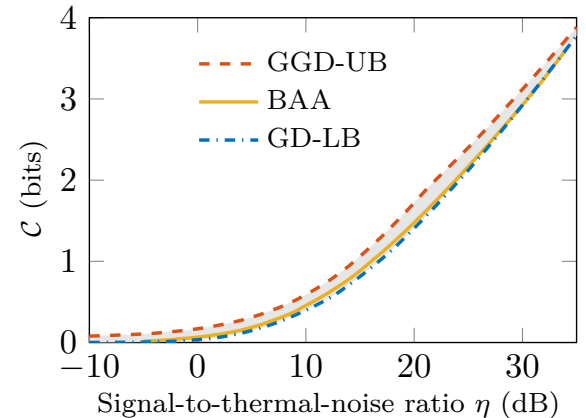

(a) $\zeta=-10 \mathrm{~dB}$

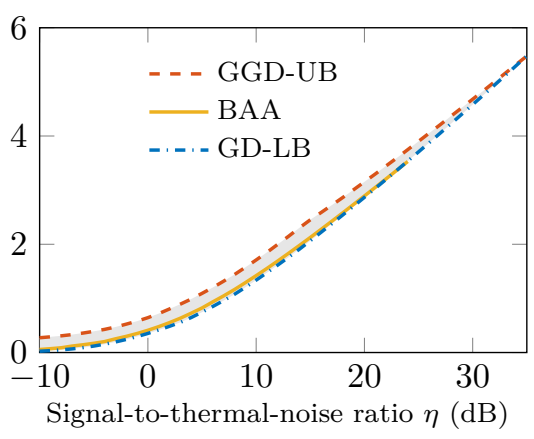

(b) $\zeta=0 \mathrm{~dB}$

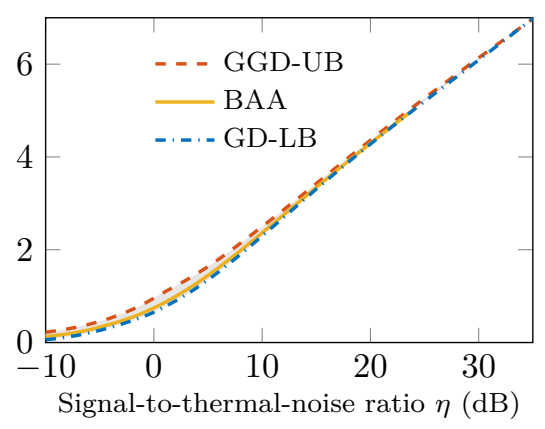

(c) $\zeta=10 \mathrm{~dB}$

Fig. 3. Bounds on the capacity for three values of thermal-to-optical-noise ratio $\zeta(6)$.

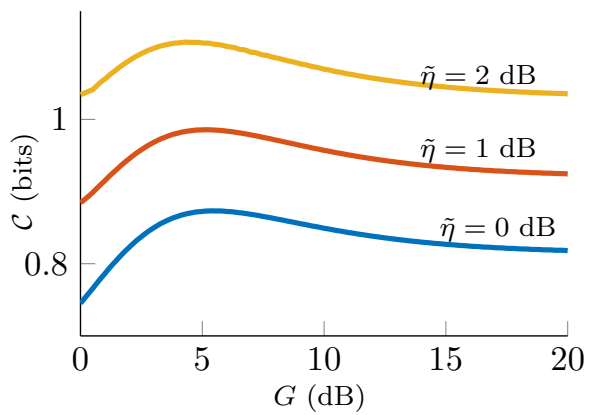

(a) $\tilde{\zeta}=5 \mathrm{~dB}$

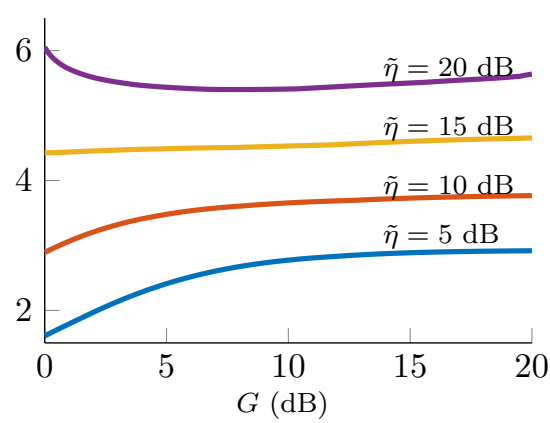

(b) $\tilde{\zeta}=15 \mathrm{~dB}$

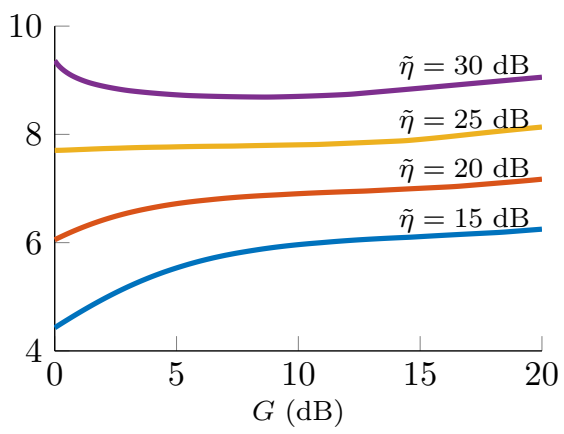

(c) $\tilde{\zeta}=25 \mathrm{~dB}$

Fig. 4. Capacity lower bounds (GD-LB) vs. the amplifier gain for several values of nonamplified-signal-to-thermal-noise ratio $\tilde{\eta}(8)$ and thermal-to-nonamplified-optical-noise ratio $\tilde{\zeta}(9)$.

increases with $\zeta$ if $\eta$ is fixed, which increases the capacity; this can be observed in Fig. 3 .

\section{DEPENDENCE ON OPTICAL AMPLIFICATION GAIN}

In this section, we study the effects of the optical amplification gain on the capacity of amplified noncoherent links (7). Fig. 4 represents the capacity lower bounds (GD-LB) for amplification gains $G$ between 0 and $20 \mathrm{~dB}$, multiple values of $\tilde{\eta}(8)$, and three values of $\tilde{\zeta}(9)$. With $\tilde{\zeta}=5 \mathrm{~dB}$, it can be seen that for the considered values of $\tilde{\eta}$, the lower bounds obtain a maximum at about $G=5 \mathrm{~dB}$. However, with both $\tilde{\zeta}=15$ and $\tilde{\zeta}=25 \mathrm{~dB}$, one can see that the lower bounds have a maximum at $G=1$ (no amplification) for large values of $\tilde{\eta}$ or are monotonically increasing with $G$ for small $\tilde{\eta}$ values.

In Fig. 5, the optimal value of $G$ is presented for a large range of parameters $\tilde{\eta}$ and $\tilde{\zeta}$. As can be seen, with low values of nonamplified-signal-to-thermal-noise ratio $\tilde{\eta}$ and thermal-to-nonamplified-optical-noise ratio $\tilde{\zeta}$, amplification with a finite value of $G$ is optimal. However, for a large range of parameters either no amplification $(G=0 \mathrm{~dB})$ or high-gain amplification maximizes the capacity. For high values of $\tilde{\zeta}$ (and high $\tilde{\eta}$ ), the borderline between these two regions can be approximated by $\tilde{\eta}(\mathrm{dB}) \approx \tilde{\zeta}(\mathrm{dB})+3$, or equivalently $\tilde{\eta}=2 \tilde{\zeta}$ (or $\tilde{P}=2 P_{\mathrm{t}} / \tilde{P}_{\mathrm{o}}$ ). As an example, if we consider an erbium-doped fiber amplifier (EDFA) with $n_{\mathrm{sp}}=2$ and also assume $\xi=1, W_{\mathrm{o}}=W_{\mathrm{t}} / 2=50 \mathrm{GHz}$,

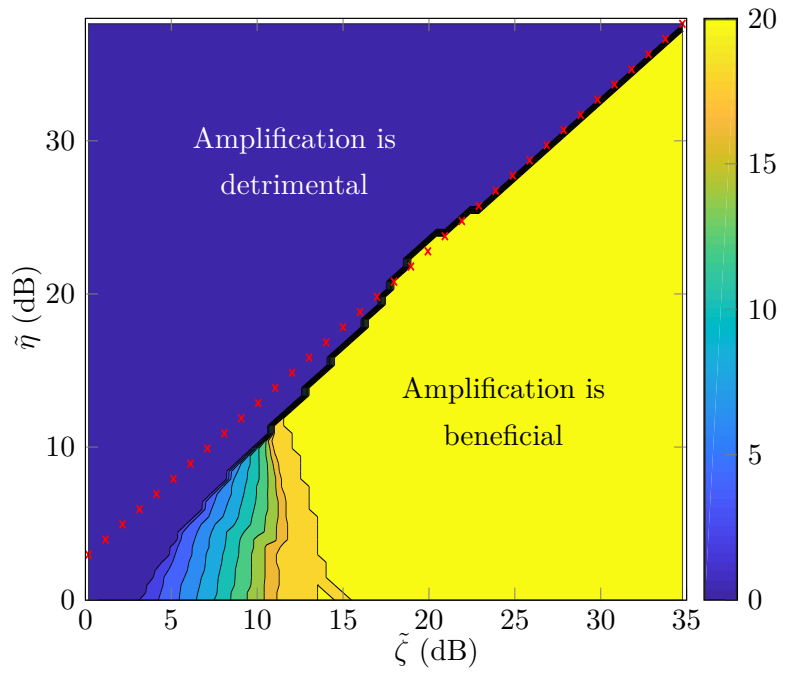

Fig. 5. Optimal amplification gains for different values of nonamplified-signal-to-thermal-noise ratio $\tilde{\eta}$ (8) and thermal-tononamplified-optical-noise ratio $\tilde{\zeta}(9)$. An approximated borderline $\tilde{\eta}(\mathrm{dB})=\tilde{\zeta}(\mathrm{dB})+3$ is also plotted (with " $\mathrm{x}$ " markers).

$T=300 \mathrm{~K}$, and $R_{\mathrm{L}}=50 \Omega$, we obtain from $(10) \tilde{\zeta} \approx 25.6$ dB. For such a system, optical amplification enhances the capacity if $\tilde{P}$ is less than $6.2 \mathrm{dBmA}(\tilde{\eta}<28.6 \mathrm{~dB})$. In general, the value of $\tilde{\zeta}$ for typical systems with EDFA is high (roughly 25-30 dB). Nonetheless, we consider the 


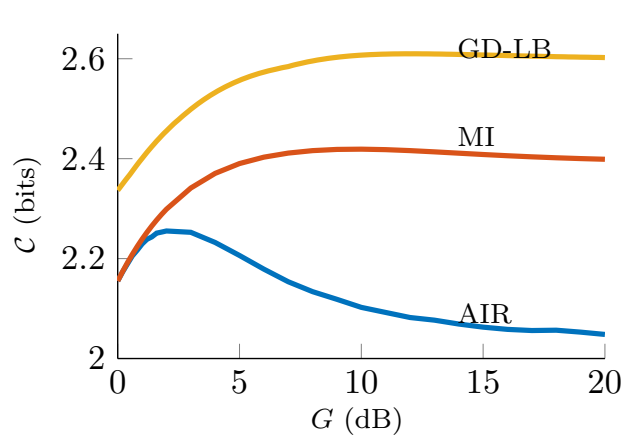

(a) $\tilde{\eta}=8 \mathrm{~dB}$

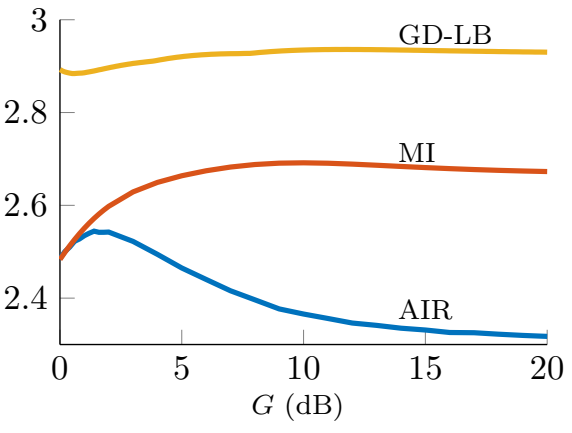

(b) $\tilde{\eta}=10 \mathrm{~dB}$

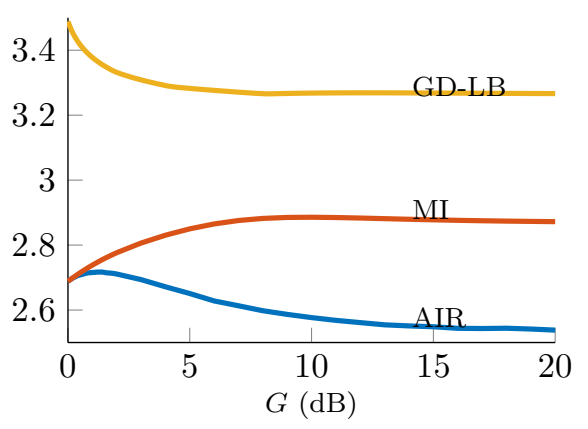

(c) $\tilde{\eta}=12 \mathrm{~dB}$

Fig. 6. Capacity lower bounds vs. amplifier gain for thermal-to-nonamplified-optical-noise ratio $\tilde{\zeta}=10 \mathrm{~dB}$ and three values of nonamplifiedsignal-to-thermal-noise ratio: $\tilde{\eta}=8 \mathrm{~dB}, \tilde{\eta}=10 \mathrm{~dB}$, and $\tilde{\eta}=12 \mathrm{~dB}$. The lower bounds include AIR for 8-PAM and an AWGN auxiliary channel, MI for 8-PAM, and GD-LB.

capacity for the whole range of $\tilde{\zeta}$ in Fig. 5, since the parameter range that is not relevant now or for a specific application might become relevant in the future or for other IMDD applications.

Finally, in Fig. 6, we compare the information rate obtained based on an 8-PAM input with our lower bound for three values of $\tilde{\eta}$. We assume transmission of equidistant symbols $(\mathrm{x} \in\{0, \ldots, 7\} \cdot \sqrt{2 P / 35})$ with uniform probabilities. The AIR calculation is based on 8-PAM and an additive white Gaussian noise auxiliary channel (see [38, Eq. (6)]), which corresponds to a suboptimal minimumdistance receiver. The information rate for 8-PAM and the optimal receiver is presented by calculating the MI $I(\mathbf{x} ; \mathbf{y})$. Furthermore, the GD-LB is provided for comparison. As can be seen in Fig. 6, different performance behaviors are observed for these three information rates. This indicates that the optimal value of $G$ can be different for suboptimal receivers and/or suboptimal input distributions compared to the one calculated based on the system capacity in Fig. 5.

\section{Conclusions}

We quantified the optimal amplification gain, which maximizes the capacity of short-haul noncoherent links, for a wide range of channel parameters. Our results indicate that with large values of the thermal-to-nonamplifiedoptical-noise ratio $\tilde{\zeta}$, either no amplification or high-gain amplification is optimal. As a rule of thumb, systems that work in this regime (e.g, typical IMDD fiber-optic systems with EDFA amplification), should deploy optical amplification at low received powers $\left(\tilde{\eta}<2 \tilde{\zeta}\right.$ or $\left.\tilde{P}<2 P_{\mathrm{t}} / \tilde{P}_{\mathrm{o}}\right)$. With relatively low values of $\tilde{\zeta}$, it was illustrated that finite optical amplification gains may maximize the capacity. Furthermore, we show that the gain that maximizes the capacity may not be optimal for a system with a suboptimal receiver or input distribution. These results were obtained by establishing bounds that characterize the capacity in the presence of both optical and thermal noises, which generalizes known results that consider either optical or thermal noise, but not both.

\section{ACKNOWLEDGMENT}

The authors gratefully acknowledge helpful discussions with Giuseppe Durisi, Enrico Forestieri, and Derek Nesset.

\section{REFERENCES}

[1] J. M. Kahn and J. R. Barry, "Wireless infrared communications," Proceedings of the IEEE, vol. 85, no. 2, pp. 265-298, Feb. 1997.

[2] M. A. Khalighi and M. Uysal, "Survey on free space optical communication: A communication theory perspective," IEEE Commun. Surveys Tuts., vol. 16, no. 4, pp. 2231-2258, 2014.

[3] G. P. Agrawal, Fiber-Optic Communication Systems, 4th ed. John Wiley \& Sons, 2010.

[4] E. Agrell et al., "Roadmap of optical communications," J. Opt., vol. 18, no. 6, May 2016, Art. no. 063002.

[5] A. Mecozzi and M. Shtaif, "Information capacity of direct detection optical transmission systems," J. Lightw. Technol., vol. 36, no. 3, pp. 689-694, Feb. 2017.

[6] E. Forestieri and M. Secondini, "On the error probability evaluation in lightwave systems with optical amplification," J. Lightw. Technol., vol. 27, no. 6, pp. 706-717, Mar. 2009

[7] M. Franceschini, G. Bongiorni, G. Ferrari, R. Raheli, F. Meli, and A. Castoldi, "Fundamental limits of electronic signal processing in direct-detection optical communications," J. Lightw. Technol., vol. 25, no. 7, pp. 1742-1753, Jul. 2007.

[8] S. Hranilovic, "Minimum bandwidth Nyquist and root-Nyquist pulses for optical intensity channels," in IEEE Global Telecommun. Conf., St. Louis, MO, USA, Nov. 2005.

[9] - "Minimum-bandwidth optical intensity Nyquist pulses," IEEE Trans. Commun., vol. 55, no. 3, pp. 574-583, 2007.

[10] M. Tavan, E. Agrell, and J. Karout, "Bandlimited intensity modulation," IEEE Trans. Commun., vol. 60, no. 11, pp. 34293439, Nov. 2012.

[11] C. B. Czegledi, M. R. Khanzadi, and E. Agrell, "Bandlimited power-efficient signaling and pulse design for intensity modulation," IEEE Trans. Commun., vol. 62, no. 9, pp. 3274-3284, Sep. 2014.

[12] K.-P. Ho, Phase-Modulated Optical Communication Systems. Springer, 2005.

[13] A. Mecozzi and M. Shtaif, "On the capacity of intensity modulated systems using optical amplifiers," IEEE Photon. Technol. Lett., vol. 13, no. 9, pp. 1029-1031, 2001.

[14] W. Mao and J. M. Kahn, "Lattice codes for amplified directdetection optical systems," IEEE Trans. Commun., vol. 56, no. 7, pp. 1137-1145, Jul. 2008.

[15] K.-P. Ho, "Exact evaluation of the capacity for intensitymodulated direct-detection channels with optical amplifier noises," IEEE Photon. Technol. Lett., vol. 17, no. 4, pp. 858860, Apr. 2005.

[16] M. Katz and S. Shamai (Shitz), "On the capacity-achieving distribution of the discrete-time noncoherent and partially coherent AWGN channels," IEEE Trans. Inform. Theory, vol. 50, no. 10, pp. 2257-2270, Oct. 2004. 
[17] G. Durisi, "On the capacity of the block-memoryless phase-noise channel," IEEE Commun. Lett., vol. 16, no. 8, pp. 1157-1160, Aug. 2012.

[18] A. Lapidoth, "On phase noise channels at high SNR," in Proc. IEEE Inf. Theory Workshop, Bangalore, India, Oct. 2002.

[19] R. You and J. M. Kahn, "Capacity bound of optical IM/DDchannels using multiple-subcarrier modulation with fixed bias," in Proc. IEEE Int. Conf. Commun. (ICC), Helsinki, Finland, Jun. 2001, pp. 2757-2762.

[20] J. Karout, E. Agrell, K. Szczerba, and M. Karlsson, "Optimizing constellations for single-subcarrier intensity-modulated optical systems," IEEE Trans. Inform. Theory, vol. 58, no. 7, pp. 46454659, Jul. 2012.

[21] R. You and J. M. Kahn, "Upper-bounding the capacity of optical IM/DD channels with multiple-subcarrier modulation and fixed bias using trigonometric moment space method," IEEE Trans. Inform. Theory, vol. 48, no. 2, pp. 514-523, Feb. 2002.

[22] S. Hranilovic and F. R. Kschischang, "Capacity bounds for power-and band-limited optical intensity channels corrupted by Gaussian noise," IEEE Trans. Inform. Theory, vol. 50, no. 5, pp. 784-795, May 2004.

[23] A. Lapidoth, S. M. Moser, and M. A. Wigger, "On the capacity of free-space optical intensity channels," IEEE Trans. Inform. Theory, vol. 55, no. 10, pp. 4449-4461, Oct. 2009

[24] A. A. Farid and S. Hranilovic, "Capacity bounds for wireless optical intensity channels with Gaussian noise," IEEE Trans. Inform. Theory, vol. 56, no. 12, pp. 6066-6077, Dec. 2010.

[25] - "Capacity of optical intensity channels with peak and average power constraints," in Proc. IEEE Int. Conf. Commun. (ICC), Dresden, Germany, Jun. 2009.

[26] S. M. Moser, M. Mylonakis, L. Wang, and M. Wigger, "Asymptotic capacity results for MIMO wireless optical communication," in Proc. IEEE Int. Symp. Inform. Theory, Aachen, Germany, Jun. 2017, pp. 536-540.

[27] S. M. Moser, L. Wang, and M. Wigger, "Capacity results on multiple-input single-output wireless optical channels," IEEE Trans. Inform. Theory, vol. 64, no. 11, pp. 6954-6966, Nov. 2018.

[28] L. Li, S. M. Moser, L. Wang, and M. Wigger, "On the capacity of MIMO optical wireless channels," in IEEE Info. Theory Workshop (ITW), Guangzhou, China, Nov. 2018.

[29] "The MISO free-space optical channel at low and moderate SNR," in 52nd Annual Conf. on Inform. Sciences and Syst. (CISS), Princeton, NJ, USA, Mar. 2018.

[30] A. Lapidoth and S. M. Moser, "Capacity bounds via duality with applications to multiple-antenna systems on flat-fading channels," IEEE Trans. Inform. Theory, vol. 49, no. 10, pp. 2426-2467, Oct. 2003.

[31] R. Blahut, "Computation of channel capacity and ratedistortion functions," IEEE Trans. Inform. Theory, vol. 18, no. 4, pp. 460-473, Jul. 1972.

[32] S. Arimoto, "An algorithm for computing the capacity of arbitrary discrete memoryless channels," IEEE Trans. Inform. Theory, vol. 18, no. 1, pp. 14-20, Jan. 1972.

[33] C. Shannon, "A mathematical theory of communication," Bell Syst. Tech. J., vol. 27, pp. 379-423/623-656, Jul.-Oct. 1948.

[34] G. P. Agrawal, Fiber-Optic Communication Systems, 4th ed. John Wiley \& Sons, 2010.

[35] N. A. Olsson, "Lightwave systems with optical amplifiers," J. Lightw. Technol., vol. 7, no. 7, pp. 1071-1082, Jul. 1989.

[36] T. M. Cover and J. A. Thomas, Elements of Information Theory. Wiley-Interscience, 1991

[37] K. Keykhosravi, G. Durisi, and E. Agrell, "Accuracy assessment of nondispersive optical perturbative models through capacity analysis," Entropy, vol. 21, no. 8, pp. 5532-5543, Aug. 2019.

[38] M. Secondini and E. Forestieri, "Scope and limitations of the nonlinear Shannon limit," J. Lightw. Technol., vol. 35, no. 4, pp. 893-902, Apr. 2017. 